\title{
Standardization of Genetic Tests in Celiac Disease
}

\author{
Iulia Lupan ${ }^{1}$, Anamaria Girbovan ${ }^{2, *}$, Gabriel Samasca ${ }^{3}$ \\ ${ }^{1}$ Department of Molecular Biology, “Babes-Bolyai” University, Cluj-Napoca, Romania \\ ${ }^{2}$ Department of Immunology, "Iuliu Hațieganu” University of Medicine and Pharmacy, Cluj-Napoca, Romania \\ ${ }^{3}$ Emergency Hospital for Children, Cluj-Napoca, Romania \\ *Corresponding author: anne.garbovan@gmail.com
}

\begin{abstract}
With all the advancements of science, genetic tests are important for the diagnosis of celiac disease but a lack of standardization is evidenced.
\end{abstract}

Keywords: celiac disease, HLA, diagnosis

Cite This Article: Iulia Lupan, Anamaria Girbovan, and Gabriel Samasca, "Standardization of Genetic Tests in Celiac Disease.” International Journal of Celiac Disease, vol. 5, no. 2 (2017): 48-49. doi: 10.12691/ijcd-5-2-9.

\section{Introduction}

Human Leucocyte Antigens (HLA) are glycoproteins present on cell membranes. These antigenes are responsible for recognition of self and non-self structures, the immune response to antigenic stimulations, and the coordination of humoral and cellular immune response. Major histocompatibility complex (MHC) molecules play a central role in intra- and inter-cellular events leading to immune response. After their synthesis and expression, on antigen-presenting cells (APC) and other cells expressing HLA, MHC molecules are brought into contact with antigens.

- peptides of intracellular origin are transported by Class I molecules;

- extracellular proteins are processed to small peptides, which are afterwards transported on cell surface Class II molecules.

On cell surface, MHC molecules influence intercellular communication for afferent and efferent components of immune response.

\section{Detection of HLA Antigens}

Reactions based on DNA using molecular biology have several advantages over serological and cellular reactions: specificity and sensitivity, requiring a lower volume of samples studied, demanding less time of processing. Another advantage of great value is not requiring the presence of cell surface antigens or cell viability.

\subsection{RFLP $=$ Restriction Fragment Length Polimorfism}

DNA isolated from peripheral blood leukocytes is fragmented into small fragments at specific positions, by specific bacterial enzymes called endonucleases. These fragments have different molecular weights will migrate on gel electrophoresis in different positions. Then, DNA is transferred from gel to a membrane. Application of labeled samples allows detection for different bands, where the sample is attached to specific segments of nucleic acid.

RFLP is being replaced today by PCR.

\section{2. $\mathrm{PCR}=$ Polymerase Chain Reaction}

a) $\mathrm{PCR}-\mathrm{SSOP} / \mathrm{SSO}=$ sequence specific oligonucleotide probes

Specific oligonucleotids hybridization generates the PCR product, which is afterwards applied to a membrane or a filter that are applied to SSOP labels. These short fragments of DNA will hybridize with complementary sequences, as a result, identifying groups or individual alleles.

The advantages are that all loci of class II can be printed in order to achieving highly specific information. Disadvantages include potential difficulties in interpreting the results and the need to use multiple filters and perform several hybridizations.

b) PCR-SSP = sequence specific primers

The method uses specific sequences of primers, targeting a particular DNA sequence. The procedure, know as PCR-SSP is based on the application of high specificity while amplifying rather than upon hybridization. Because SSP has some specific targets, the presence of amplified material indicates the corresponding alleles.

SSOP and SSP can be combined resulting in highresolution typing. Low resolution typing or generic detects equivalence HLA serology with high accuracy, distinguishing the DR2 by DR1, while high-resolution typing distinguishes individual alleles, and the DRB * 0101 by DRB * 0102 .

\section{Conclusion}

Standardization of genetic tests is needed in celiac disease diagnosis. 


\section{References}

[1] Dyer P., Middleton D. Histocompatibility Testing, A Practical Approach. Oxford University Press; 1992.
[2] Inoko H. and Ota M. PCR-RFLP, in Handbook of HLA Typing Techniques (Hui K.M. and Bidwell J.L. et all) CRC Press, Boca Raton;1993:9-70. 